\title{
REVISION DE LAS ESPECIES DE LA FAMILIA LICHINACEAE INCLUIDAS EN EL HERBARIO WERNER (BC)
}

\author{
Pedro P. MORENO \& José M. EGEA
}

RESUMEN: Recopilamos en este estudio todas las citas de especies de la familia Lichinaceae incluidas en la extensa bibliografía sobre el norte de Africa del Prof. R.G. Werner y revisamos los pliegos correspondientes conservados en el herbario del Instituto Botánico de Barcelona (BC).

Palabras clave: Líquenes, liquinaceas, taxonomía, norte de Africa.

ABSTRACT: All species of the lichen family Lichinaceae reported from northern Africa in Prof. Werner's works are compiled. A revision on the material collected corresponding to these species and actually included in BC herbarium is made.

Key words: Lichens, lichinaceae, taxonomie, northern Africa.

\section{N T R O D U C C I O N}

La parte más prolífica de la producción científica del Profesor R.G Werner tuvo lugar durante su período de estancia en Marruecos, a cargo del Instituto Científico Chérifien de Rabat y de la Estación de Biología Vegetal del Atlas Medio, entre los años 1929 y 1948, aunque los resultados de sus estudios en el norte de Africa continuaron saliendo a la luz largo tiempo después.

Dentro de esta producción y en lógica correspondencia con el tratamiento que se concede a la familia Lichinaceae en la liquenología en general, las especies de la misma citadas son relativamente pocas. Un total de 20, a las que hay que sumar 2 variedades, aparecen reflejadas en su extensa bibliografía. Entre ellas, 6 especies y 2 géneros fueron descritos como nuevos para la ciencia. Esto supone un catálogo exiguo, pero de una gran importancia si tomamos en consideración la casi total ausencia de trabajos previos, sobre todo en Marruecos, donde los estudios de Bouly de Lesdain (1924) y Szatala (1931) son prácticamente lo único a reseñar y en los que aparecen citadas un total de cuatro especies: Synalissa symphorea y Lempholemma moureti en el primero de ellos y Peccania coralloides y Psorotichia murorum en el segundo.

Realizado en el marco del Proyecto de Investigación Nr 0666-84 subvencionado por la C.A.I.C.Y.T. 
El escaso eco despertado por los trabajos de este autor, la importancia de sus citas en lo que a Lichinaceae se refiere por ser, junto al catálogo de Flagey (1897) en Argelia, la base para ulteriores estudios, y el interés que supone la posibilidad de establecer comparaciones biogeográficas con la flora del sureste español, nos ha llevado a revisar todo el material de este grupo incluido en su herbario, dentro del marco de nuestro estudio sobre la familia Lichinaceae en el sureste de la Península Ibérica y norte de Africa.

\section{R E S UL T A D OS}

El herbario Werner, conservado en la actualidad en el Instituto Botánico de Barcelona (BC), incluye un total de 43 ejemplares de la familia Lichinaceae. En el listado que exponemos a continuación tomamos como base las citas bibliográficas del autor para, a partir de ahí, exponer nuestras propias observaciones sobre el material correspondiente.

1.- Anema decipiens (Massal.) Forss.

Citas: MARRUECOS, Meknes, Macizo de Zerhoun, descenso del Col de Segotta hacia Fez; (Werner, 1970).

Observaciones: El material incluido en el herbario correspondiente a esta cita es, en realidad, Anema nummularium (Duf.) Nyl. Se trata de un conjunto de talos jóvenes, escuamuloso-umbilicados, carentes de pruina, netamente lobulados en la periferia y con numerosos ascocarpos de disco pardo-rojizo por escuámula, caracteres todos ellos que confirman nuestra determinación.

Mezclado con este material aparece un único talo, mal conservado, que corresponde a Thyrea girardi (Dur. et Mont.) Bagl. et Car.

\section{2.- Gonohymenia monicae Wern.}

Citas: MARRUECOS, Macizo de Zerhoun, Col de Segotta cerca de las ruinas de la ciūdad romana de Volubilis; (Werner, 1970).

Observaciones: No hemos podido estudiar el material tipo de esta especie, dado que se encuentra prestado a la Prof. A. Henssen. En carta dirigida por la citada investigadora al Dr. Llimona (com. pers.) el ejemplar corresponde a Sarcogine pruinosa auct.

\section{3.- Lempholemma moureti B. de Lesd.}

Citas: Sin localidad (Werner, 1931; probablemente tomada de Bouly de Lesdain, 1924).

Observaciones: No hemos hallado en BC material correspondiente a esta cita.

\section{4.- Lichina pygmaea (Lighft.) Agardh}

Citas: MARRUECOS. Playa de Miramar, $10 \mathrm{Km}$ al sur de Rabat; (Werner, 1932). Sobre las rocas costeras, cerca de la desembocadura del Oued Ykem, Fedala; (Werner, 1936). Isla de Mogador; (Werner, 1938), Rabat. Playa de Temara. Oued Ykem. Fedala-Oued NefifikOued Mellah. Dar bou Azza- Dar ben Abid. Mazagan-Azemmour. Mogador. Bahía de Imsaouane. Norte del Cabo Rhir. El Khemis (N de Agadir) y Agadir; (Werner, 1962).

Observaciones: Tampoco de esta especie hemos hallado pliego alguno correspondiente a las citas bibliográficas anteriores. No obstante, podemos constatar la presencia de L. pygmaea en la costa atlántica marroquí en función de nuestras propias observaciones (Moreno, 1988), 
además, la presencia de varias exsiccatae del taxon procedentes de otros herbarios en la colección del Prof. Werner indica que este autor debía conocerlo a fondo (ver lista de exsiccatae al final).

\section{5.- Lichinella stipatula $\mathrm{Nyl}$.}

Citas: MARRUECOS, Mont Kebir en la región de Tanger; (Werner, 1939).

Observaciones: Dos ejemplares aparecen marcados como L. stipatula procedentes del lugar citado más arriba. En el primero de ellos encontramos esta especie mezclada con una del género Spilonema en estado estéril; en el segundo aparece sólo esta última especie y no hay rastro de L. stipatula.

Otra colección de este taxon aparece acompañando al ejemplar de Tingiopsidium pubescens Wern. en la misma localidad.

La distribución de L. stipatula en Marruecos es mucho más amplia de lo que parece indicar esta única cita, siendo muy frecuente sobre sustratos ácidos en el Alto Atlas y en el AntiAtlas (Moreno, 1988).

\section{6.- Peccania coralloides Massal.}

Citas: MARRUECOS. Sobre calizas lacustres en la región de Saïs, entre Fez y Meknes, 500 m.s.m. Grutas de Hércules al sur de Mont Kebir en Tanger; (Werner, 1939). Oued Zmoul. Kheneg bou Mrheinfra; (Werner, 1954). Cabo Rhir a $46 \mathrm{Km}$ al norte de Agadir, sobre acúmulos de tierra entre las rocas; (Werner, 1955a). Mont Bani cerca de Foum Zguid. Bajo Dra: territorio en el perímetro de Kheneg-el-Haman, Aouriora, Cabo Dra y El Ayoun (Werner, 1963). Marrakech en el Mont Gueliz. Región de Assa, Djebel Targoumaït; (Werner, 1967).

Observaciones: A pesar del número de citas indicadas, el material de esta especie incluido en el herbario es bastante escaso y heterogéneo.

El material correspondiente a la primera cita indicada (región de Saïs) es, en efecto, $P$. coralloides, su himenio maculado, su morfología y tamaño esporal y sus conidios filiformes así lo indican. No obstante, los ejemplares muestran sus lóbulos aplanados en lugar de cilíndricos como en el lectotypus de la especie. Esto ya ha sido discutido anteriormente (Henssen \& Büdel, 1984; Moreno, 1988) y, al parecer, se trata de un morfotipo muy frecuente en el área mediterránea española y norteafricana.

Por contra, el resto del material incluido como Peccania coralloides, correspondiente a las localidades del Bajo Dra, Marrakech y Djebel Bani, no es sino Heppia turgida (Ach.) Nyl.

\section{7.- Peccania coralloides Massal. var. arenicola Hue}

Citas: MARRUECOS. Sobre tierra, en un barranco entre Kreb e Igman, en el borde meridional de la Hammada del Dra; (Werner, 1954).

Observaciones: Las características de este ejemplar coinciden bastante bien con las indicadas por Hue (1897) en la descripción original del taxon. No obstante, P. tiruncula (Nyl.) Hens., es una especie muy próxima, con la que abría que compararla. Lamentablemente, no hemos podido disponer del tipo de $P$. coralloides var. arenicola para su estudio por lo que, de momento, preferimos mantener la determinación del Profesor Werner.

\section{8.- Peccania synaliza (Ach.) Forss.}

Citas: CORCEGA, Caporalino, en el monte Pollino, sobre los muros en el interior de una torre en ruinas. (Werner \& Deschatres, 1970).

Observaciones: El ejemplar corresponde a Synalissa symphorea (Ach.) Nyl.

\section{9.- Psorotichia isidiosa Wern.}

Citas: MARRUECOS. Sobre esquistos, cerca de Bouznika, en la ruta de Rabat a Casablanca; (Werner, 1975).

Observaciones: En el herbario hemos encontrado dos pliegos marcados como $P$. isidiosa. Uno de ellos contiene un ejemplar de Pyrenopsis cuyos caracteres lo aproximan a $P$. triptococca Nyl. En el otro pliego encontramos dos especies diferentes, una que podría corresponder a la especie del caso anterior pero en peor estado (son muestras de la misma localidad: 
Monts Zaïans, Gargantas de Tabarat a $10 \mathrm{Km}$ de Harcha); y otra cuyas características, recolector y localidad coinciden con los datos del protólogo, excepto por encontrarse en estado estéril. Esta segunda especie es, en realidad, Lichinella stipatula Nyl., representada por numerosas almohadillas de talos jóvenes. La presencia de L. stipatula invadiendo los talos de Pyrenopsis, la ausencia de fructificaciones en el caso de la primera especie, la coincidencia de caracteres himeniales y esporales entre este Pyrenopsis y las indicadas en el protólogo, y la aparición en el mismo pliego de muestras de localidades diferentes nos llevan a pensar en una descripción errónea de $P$. isidiosa, combinando los caracteres de las otras dos especies citadas.

Por todo ello, hemos elegido como lectotypus de $P$. isidiosa Wern. el material correspondiente a la localidad indicada en el protólogo y lo emplazamos como sinónimo de Lichinella stipatula $\mathrm{Nyl}$.

En el mismo sobre aparece también Peltula euploca, especie que siempre acompaña sobre rocas ácidas a $L$. stipatula y junto a las que se desarrolla ocasionalmente $P$. aff. triptococc $a$, constituyendo un grupo de especies muy frecuente en el sureste de España y norte de Africa.

10.- Psorotichia murorum Massal.

Citas: MARRUECOS, Bas Dra: territorio en el perímetro de Kheneg-el Haman, Aouriora, Cabo Dra y El Ayoun; (Werner, 1963).

Observaciones: Los dos ejemplares marcados como $P$. murorum fueron recolectados en la Hammada entre Aït ben Haddou y Tazenakht, en el año 1935, por lo que no corresponden a la cita bibliográfica reseñada más arriba.

El primero de ellos contiene un ejemplar mal desarrollado de $P$. murorum caracterizada por su talo de superficie irregular o débilmente granulosa, con 1-4 ascocarpos por escuámula, apotecios prominentes, provistos de un grueso excípulo propio de $10-15 \mu \mathrm{m}$, ascos octosporados y ascósporas elipsoidales de 9-16 x 7-9 $\mu \mathrm{m}$. Sobre el mismo fragmento de roca aparece un talo fértil, aunque poco desarrollado, de Gonohymenia nummularia (Nyl.) Henss.

El segundo pliego corresponde a Peccania tiruncula (Nyl.) Hens. por su talo de desarrollo peltado, ascocarpos con disco pardo amarillento, himenio maculado, ascos octosporados provistos de vaina gelatinosa engrosada apicalmente y conidios filiformes.

11.- Psorotichia ocellata (Th. Fr.) Forss.

Citas: MARRUECOS, Col de Segotta cerca de Fez en exposición sudeste sobre calizas lacustres, 500 m.s.m.; (Werner, 1970).

Observaciones: Esta especie se caracteriza por su talo crustáceo formado por diminutas granulaciones verruciformes más o menos aisladas, con ascocarpos puntiformes en origen y urceolados al final de su desarrollo. Un carácter diagnóstico sencillo de observar y que se muestra absolutamente constante en todo el material examinado en España y en el norte de Africa (Moreno, 1988) es la presencia de un epihimenio verde-azulado.

\section{2.- Psorotichia schaereri (Massal.) Arnold}

Citas: MARRUECOS, Tanger, Mont Kebir; (Werner, 1939).

Observaciones: No hemos encontrado en BC el material correspondiente a esta cita. Sí aparecen, sin embargo, dos sobres procedentes de Egipto (El Cairo, a $20 \mathrm{Km}$ en el Djebel Asfar) y marcados como P. schaereri var. arenaria (Arn.) Forss. En ambos casos se trata de ejemplares en muy mal estado de conservación, que no hemos podido estudiar convenientemente.

13.- Psorotichia suffugiens (Nyl.) Forss.

Citas: MARRUECOS, $17 \mathrm{Km}$ al norte de Agadir en la ruta de Mogador; (Werner, 1975).

Observaciones: Existen en BC dos ejemplares de esta especie bien caracterizada por su morfología talina y sus ascos multisporados.

Al ser la única especie del género que posee ascos multiesporados su identificación es relativamente sencilla, aunque se relaciona muy estrechamente con Pterygiopsis affinis 
(Massal.) Hens. de la que se separa por no presentar talo placoide fisurado-areolado y por el mayor tamaño de sus ascosporas.

\section{4.- Psorotichiella davidis Wern.}

Citas: EGIPTO, cerca de la fuente Qastal el Moaf, en el Baer, en la ruta de Antioquía a Lattaquia a $40 \mathrm{Km}$ de esta villa; (Werner, 1955 b).

Observaciones: El material correspondiente al lectotypus elegido por nosotros es muy fragmentario y en todos los casos presenta esporas fuertemente colapsadas, lo que dificulta su estudio. En cualquier caso, la presencia de esporas septadas y de un talo totalmente pseudoparenquimático lo sitúan próximo a especies del género Placynthium Gray y, por tanto, fuera de la familia Lichinaceae.

15.- Pyrenopsis lecanopsoides Nyl. var. marginata Maheu et Werner

Citas: MARRUECOS, Atlas medio, Ifrán, cercanías de la Estación de Biología Vegetal, 1600 m.s.m.; (Maheu \& Werner, 1933).

Observaciones: P. lecanopsoides Nyl. es tratado por Zahlbruckner (1924) como sinónimo de Porocyphus areolatus (Flot.) Körb. que, a su vez, se emplaza como sinónimo de $P$. coccodes (Flot) Körb. (Henssen, 1963 a). Los ejemplares estudiados corresponden a Placynthium nigrum (Huds.) Gray, aunque muestran un hipotalo poco desarrollado; pero la presencia de un neto pseudoparénquima a lo largo de todo el talo y de la típica coloración verde-azulada del mismo en la zona de contacto con el substrato calizo nos lo diferencian claramente de $P$. coccodes, especie que, aunque se da como presente en el norte de Africa (Henssen, 1963 a; Ozenda \& Clauzade, 1973), no hemos hallado en el curso de nuestras investigaciones en el área. 16.- Pyrenopsis rhodosticta (Tayl.) Müll. Arg.

Citas: MARRUECOS, Campo Bouhault al norte de Casablanca, Sokhrate-En-Nemra; (Werner, 1975).

Observaciones: Todo el material hallado corresponde a colonias de Cianofíceas no liquenizadas.

17.- Pyrenopsis subolivacea Wern.

Citas: MARRUECOS. Cheikh Abdallah en Aït-Baha, 425 m.s.m., sobre rocas calcáreas; (Werner, 1956). Ida Ou Gnidif en Aït Toudma, 1620 m.s.m. sobre cuarcitas en exposiciones norte y oeste. En el Djebel Kest, 2000-23000 m.s.m. sobre cuarcitas. Aï Abdallaj, 1600 m.s.m. sobre cuarcitas; (Werner, 1957). Gran Atlas occidental, Bled Touloun de Ida-ou-Zal; (Werner, 1963).

Observaciones: El material conservado en $\mathrm{BC}$ corresponde a la primera cita indicada, y nuestros estudios revelan que se trata de una especie de Psorotichia con talo granular, ascocarpos prominentes, urceolados, con disco pardo a rojizo, ascos octosporados y ascósporas elipsoides de 8-11 x 6-8 $\mu \mathrm{m}$. Todos estos caracteres la aproximan a P. frustulosa Anzi, aunque su estatus definitivo queda pendiente en espera de poder estudiar los tipos correspondientes.

18.- Thyrea girardi (Dur.et Mont.) Bagl. et Car.

Citas: MARRUECOS, sobre cuarcitas en la garganta del Qued Cherrat cerca de Aïn Madnet (región de Campo Bouhault): (Werner, 1935). Col de Segotta cerca de Fez, 300 m.s.m.; (Werner, 1970).

Observaciones: El material estudiado muestra el típico talo foliáceo-umbilicado, totalmente recubierto de pruina, característico de esta especie, y se encuentra sin fructificar, lo que es también extremadamente frecuente para este taxon ampliamente distribuido por el norte de Africa.

19.- Thyrea phylliscoides (Nyl.) Zahl.

Citas: MARRUECOS, Agadir; (Werner, 1975). Sin localidad (Sahara) sub Thyrea nummularioides (Nyl.) Massal.; (Emberger, Werner \& Boudy. 1934): Werner, 1967). 
Observaciones: Sólo hemos encontrado el material correspondiente a la cita de Agadir, en el que aparecen exclusivamnte talos jóvenes pero bien fructificados de Gonohymenia nummularia (Nyl.) Hens., con sus típicos talinocarpos apenas prominentes y sus ascos multisporados.

20.- Tingiopsidium pubescens Wern.

Citas: MARRUECOS, Tanger, Mont Kebir, 100 m.s.m.; (Werner, 1939).

Observaciones: Este material fue previamente estudiado por la Prof. Henssen quien lo sinonimizó con Koerberia sonomensis (Tuck.) Hens. (Henssen. 1963 b).

Aparte de todo el material expuesto anteriormente, en BC encontramos también tres pliegos marcados como Ephebe lanata (L.) Vain., especie que, según nuestros datos no llegó a aparecer publicada nunca en la obra del Prof. Werner:

\section{Ephebe lanata (L.) Vain.}

Localidad: MARRUECOS, Montes Zaians, Gargantas de Tabahart.

Observaciones: De estos pliegos, dos corresponden a la especie Thermutis velutina (Ach.) Flot. y uno a Spilonema paradoxum Born., especies que, aunque de talo filamentoso, son fácilmente separables de E. lanata.

El resto de los ejemplares incluidos en el herbario Werner, hasta completar la cifra de 43 pliegos anteriormente indicada, son exsiccatae de diferentes autores y herbarios tal y como queda a continuación:

Ephebe lanata (L.) Vain. (sub. E. pubescens)

EXSICCATA Herb. J. Maheu, Tirol, morrenas de Similhaun-Stubail. Leg. J. Maheu, VIII-1907.

\section{Lichina confinis (Müll.) Agardh}

SWEDISH LICHENS. Bohuslän: par Lagelanda. Svanesund. Leg. A.H. Magnusson 18-VII-1926.

Otros dos ejemplares de esta especie, de los que no tenemos constancia que sean exsiccatae, aparecen en el herbario, uno procedente de Holanda y otro de Francia.

\section{Lichina pygmaea (Lightf.) Agardh}

EXSICCATA Herb. J. Maheu, Córcega, Leg. J. Maheu, VIII-1909.

Synalissa symphorea (Ach.) Nyl.

LICHENES BOHEMOSLOVAKIAE, Moravia central, Brno, en el valle del río Ricky cerca de Ochoz, 350 m.s.m. Leg. A. Vezda, III-1958 (dos ejemplares).

Thyrea pulvinata (Schaer.) Massal.

EXSICCATA Herb. J. Maheu: roca calcárea cortada a pico, cerca del puente del Canal de Loing, a lo largo de la ruta de St. Mamines. Leg. Moret, 16-IV-1911.

Addenda. A continuación se relacionan los nombres correctos (en cursiva) de los táxones de la familia Lichinaceae estudiados, citados en la bibliografía del Prof. Werner. Los táxones señalados con un asterisco se encuentran depositados en el herbario BC-Werner.

* Anema nummularium (Duf.) Nyl. (sub. A. decipiens (Massal.) Forss.)

* Ephebe lanata (L.) Vain. (sub. E. pubescens Fr.)

Gonohymenia monicae Werner = Sarcogyne pruinosa auct.

* Gonohymenia nummularia (Nyl.) Henssen (sub. Thyrea phylliscoides (Nyl.) Zahlbr.)

Lempholemma moureti B. de Lesd. 
Lichina confinis Müll. Arg.

* Lichina pygmaea (Lightf.) Agardh.

* Lichinella stipatula Nyl.

* Pecania coralloides Massal.

* Peccania coralloides Massal. var. arenicola Hue

* Peccania coralloides Massal. $=$ Heppia turgida $($ Ach. $)$ Nyl.

* Peccania tiruncula (Nyl.) Henssen (sub. Porotichia murorum Massal.)

* Psorotichia isidiosa Werner $=$ Lichinella stipatula $\mathrm{Nyl}$.

* Psorotichia isidiosa Werner $=$ Pyrenopsis aff. triptococca Nyl.

* Posorotichia murorum Massal.

* Posorotichia occellata (Th. Fr.) Forss.

* Psorotichia schaereri (Massal.) Arnold var. arenaria (Arn.) Forss. (?)

* Psorotichia suffugiens (Nyl.) Forss.

* Psorotichiella davidis Werner = Placynthium $\mathrm{sp}$.

* Pyrenopsis lecanopsoides Nyl. var. marginata Maheu et Werner = Placynthium nigrum Huds.) Gray

* Pyrenopsis rhodosticta (Tayl.) Müll. Arg. = Cianofíceas no liquenizadas.

* Pyrenopsis subolivacea Werner $=$ Psorotichia frustulosa Anzi (?)

* Spilonema paradoxum Born. (sub. Ephebe lanata (L.) Vain.)

* Synalissa symphorea (Ach.) Nyl. (sub. Peccania synaliza (Ach.) Forss.)

* Thermutis velutina (Ach.) Flot. (sub. Ephebe lanata (L.) Vain.)

* Thyrea girardi (Dur. et Mont.) Bagl. et Cav.

* Thyrea pulvinata (Schaer.) Massal.

Tingiopsidium pubescens Werner = Koerberia sonomensis (Tuck.) Henssen

\section{B I B L I O G R A F I A}

BOULY DE LESDAIN, M. -1924- Lichenes du Maroc recueillis par M. Mouret en 1912. Memoires Soc. Sci. Nat. Marcoc, 8(2):290-299.

EMBERGER, L., WERNER, R.G. \& BOUDY, P. -1934- La végétation et la flore du Maroc. In: La Science au Maroc. Publié à l'ocasion de la 58ème session de l'Association Française pour l'Avancement des Sciences. Casablanca.

FLAGEY, C. -1897- Catalogue des Lichens de l'Argérie. In: Baitandier et Trabut, Flore de l'Algérie. Alger, Jordan, $139 \mathrm{pp}$.

HENSSEN, A. -1963a- Eine Revision der Flechtenfamilien Lichenaceae und Ephebaceae. Symb. Bot. Upsal. 18(1):123 pp.

HENSSEN, A. -1963b- Study of the genus Koerberia. Canadian Journal of Botany, 41:16781724.

HENSSEN, A. \& BÜDEL, B. -1984- Peccania cerebriformis und Psorotichia columnaris, zwei neue Lichinaceen von Lanzarote. Int. J. Myc. Lich., 1(3):261-271.

HUE, A. -1897- Lichenes. In: Patouillard. Catalogue raisonné des plantes cellulaires de la Tunisie. Paris.

MAHEU, J. \& WERNER, R.G. -1933- Etude sur la flore cryptogamique du Maroc I, Ann. de Crypt. exot., 7:173-194.

MORENO, P.P. -1988- Estudio de la familia Lichinaceae en el sureste de la Península Ibérica y norte de Africa. Tesis doctoral inédita. Universidad de Murcia.

OZENDA, P. \& CLAUZADE, G. -1970- Les Lichens. Etude Biologique et Flore Illustrée. Masson et Cie. Paris.

SZATALA, O. -1931- Lichenes du Maroc recueillis par M. le baron G. Andreaszky en 1930. Ungarische Bot. Blätter, 30:115-125. 
WERNER, R.G. -1932- Contribution à la flore cryptogamique du Maroc VII. Rev. Bryol. et Lichénol. 5:210-229.

WERNER, R.G. -1935- Contribution à la flore cryptogamique du Maroc XII. Bull. Soc. Sci. Nat. du Maroc, 15:267-278.

WERNER, R.G. -1936- Contribution à la flore cryptogamique du Maroc XIV. Bull. Soc. Sci. Nat. du Maroc, 16:180-190.

WERNER, R.G. -1938- Contribution à la flore cryptogamique du Maroc XVII. Bull. Soc. Sci. Nat. du Maroc, 18:126-135.

WERNER, R.G. - 1939- Contribution à la flore cryptogamique du Maroc XVIII. Etude biogéografique et écologique sur la flore lichénique de la région de Tanger. Bull. Soc. Sci. Nat. du Maroc, 19:40-54.

WERNER, R.G. -1954- Lichenes et champignons nord-africains. Rev. Bryol. et Lichénol. 23(1-2): 197-213.

WERNER, R.G. -1955a- Contribution à la flore cryptogamique du Maroc XIX. Bull. Soc. Sci. Nat. du Maroc. 35:19-67.

WERNER, R.G. -1955b- Notes de lichenologie libano-syrienne II. Bull. Soc. Bot. France, 102(7-8):349-356.

WERNER, R.G. -1956- Contribution à la flore cryptogamique du Maroc XX. Lichens de l'Anti-Atlas. Bull. Soc. Hist. Nat. Afr. du Nord., 47:84-91.

WERNER, R.G. -1957- Contribution à la flore cryptogamique du Maroc XXI. Bull. Soc. Hist. Nat. Afr. du Nord., 48:441-453.

WERNER, R.G. -1962- Essai d'une étude de la repartition des cryptogames marines et maritimes du Maroc. Bull. Soc. Sc. Nat. et Phy. du Maroc, 42:1-33.

WERNER, R.G. - 1963- Flore lichénique du Maroc méridional. Rev. Bryol. et Lichénol, 32(14):270-278.

WERNER, R.G. -1967- Lichenes et fungi maroccani II. Bull. Soc. Myc. France, 83(2): 1967.

WERNER, R.G. -1970- Lichénologie du prérif méridional et de l'ancien détroit sud-rifain environnant. Bull. Acad. et Soc. Lorraines Sci., 9(3):484-505.

WERNER, R.G. -1975- Lichens nouveaux pour le Maroc ou la science avec regard sur la Grèce. Sci. Annals of the Ach. of Agric. and Forestry. vol. Roussopoulos: 1-5.

WERNER, R.G. \& DESCHATRES, R. -1970- Nouvelle contribution à l'étude des lichens de la Corse. Bull. Soc. Bot. France, 117-261-282.

ZAHLBRUCKNER, A. -1925- Catalogus Lichenum Universalis, Vol. II. Leipzig.

(Aceptado para su publicación el 16 de enero de 1990)

Dirección de los autores: Departamento de Biología Vegetal (Botánica). Facultad de Biología. Universidad de Murcia. 30071-MURCIA. 